\title{
Motions caused by the growth of Bacillus subtilis macrofibres in fluid medium result in new forms of movement of the multicellular structures over solid surfaces
}

\author{
Neil H. Mendelson, ${ }^{1}$ Joelle E. Sarlls ${ }^{2}$ and John J. Thwaites ${ }^{3}$
}

Author for correspondence: Neil H. Mendelson. Tel: +1 520621 3617. Fax: +1 5206213709. e-mail:nhm@u.arizona.edu

1,2 Department of Molecular and Cellular Biology ${ }^{1}$ and Department of Physics², University of Arizona, PO Box 210106, Tucson, AZ 85721-0106, USA

3 Gonville and Caius College, Cambridge CB2 1TA, UK
Bacillus subtilis macrofibres, highly ordered multicellular structures, undergo twisting and writhing motions when they grow in fluid medium as a result of forces generated by the elongation of individual cells. Macrofibres are denser than the fluid medium in which they are cultured, consequently they settle to the bottom of the growth chamber and grow in contact with it. The ramifications of growth on plastic and glass surfaces were examined. Macrofibres were observed to rotate about a vertical axis near the centre of their length in a chiral-specific direction. Right-handed fibres rotated clockwise on plastic surfaces at approximately $4^{\circ} \mathrm{min}^{-1}$, left-handed structures of lower twist rotate anti-clockwise at about half that rate. Very large ball structures produced late in macrofibre formation perched on many small protruding fibres but rotated only when driven by large fibres attached to their periphery. Closer examination showed that fibres made contact with surfaces at only a few points along their length (between 1 and 6 on glass). The regions in contact with the surface changed periodically as a result of rotation of the fibre shaft caused by growth. Every time the weight of a fibre transferred from one contact point to another, each section of the fibre took a small step approximately proportional to its distance from the fibre mid-point. The net result was a rolling of each section over the surface so that the fibre rotation about a vertical axis was produced. Macrofibres also took large steps when part of the structure rose off the floor, swept through an arc in the fluid and then returned to the floor at a new location. The rate of movement during a large step, measured as the change of angle between the moving and stationary portions of the fibre, was $5^{\circ} \mathrm{s}^{-1}$. These observations reveal that the forces derived from helical growth that lead to macrofibre formation also cause characteristic macrofibre motion that differs from classical motility.

Keywords: bacterial fibres, motions on surfaces, rolling, walking, pivoting

\section{INTRODUCTION}

Bacterial macrofibres are multicellular structures produced when cylindrical cells do not separate after septation (Mendelson, 1978). Inhibition of separation in Bacillus subtilis results when the autolytic enzymes required to cleave septa are suppressed either genetically or physiologically (Fein, 1980). Starting with a single cell or spore of Bacillus subtilis the clone produced by growth without separation in a complex fluid medium initially forms a long filament with individual cells linked in tandem (Mendelson, 1976). Such filaments rotate about their long axis as they elongate, suggesting that each cell in them twists as it grows (Mendelson, 1982). Twisting filaments bend spontaneously until self or other contact is made, whereupon they supercoil by plying together. Two-ply filaments behave in the same way, producing four-ply and ultimately multi-ply structures called macrofibres, which can have hundreds or more filaments in cross section. A mature macrofibre consists therefore of a long single-cell filament containing about $10^{5}$ cells repeatedly folded upon itself and twisted together into a macroscopic fibre that is millimetres in length. 
At all stages of macrofibre formation the structures have the same helix hand and twist, suggesting that individual cells grow with constant twist in a given environment and that the supercoiling process responsible for fibre morphogenesis is always negative supercoiling (Mendelson et al., 1984). Macrofibre helix hand and degree of twist are governed by both genetic and environmental factors. Many strains are capable of forming structures ranging from tight right-handed to tight left-handed fibres depending upon environmental conditions (such as temperature, nutrient composition and the concentration of certain ions) and can be made to change from one form to another by changing the environment (Mendelson \& Thwaites, 1988). Although macrofibre helix hand and twist state are thought to reflect conformation states of the cell wall polymers, presumably established during peptidoglycan incorporation into the fabric of the wall, a direct relationship between polymer and cellular structure remains to be shown.

The observation that macrofibres sediment when transferred into fresh fluid medium of the same composition as that in which they are produced suggests that fibre morphogenesis occurs on the floor of the growth chamber rather than in the bulk phase of the fluid growth medium. If so, the contact fibres make with the floor is likely to be a significant factor in fibre assembly because impediment of the twisting produced when cells grow triggers supercoiling, the key process in fibre morphogenesis. Macrofibres on a surface may be influenced in two ways: by friction and by the constraining effect of the surface. Whether friction or boundary effects do indeed influence macrofibres growing on a surface was sought in the experiments described here.

To examine fibre-surface interactions, a new microscopy system was constructed that provides both a lateral view of fibres and the floor of a glass growth chamber, as well as a conventional view of the structures from above. Analysis of video films obtained using the new system show that fibres make contact with the floor during their growth and undergo motions such as rotation about a centre located near the centre of a fibre's length, rolling over the surface and stepping. In addition, fibres assume configurations not previously observed when they were viewed either from above or below: they perch on points such as the loops at their ends or side branches that protrude from the fibre shaft.

\section{METHODS}

Strains. B. subtilis 168 strains FJ7 and RHX have been described previously (Mendelson, 1978; Mendelson \& Favre, 1987). Both are capable of growing as either left- or righthanded macrofibres with a range of twist states. Young fibres produced in standard fluid cultures were used as the starting material for all experiments. FJ7 fibres were used to study the behaviour of right-handed structures, RHX fibres to study left-handed forms.

Media and growth conditions. The complex medium TB, consisting of $10 \mathrm{~g}$ Bacto Tryptose (Difco), $3 \mathrm{~g}$ Bacto Beef Extract (Difco) and $5 \mathrm{~g} \mathrm{NaCl}$ (1 deionized water) ${ }^{-1}$ (Mendelson

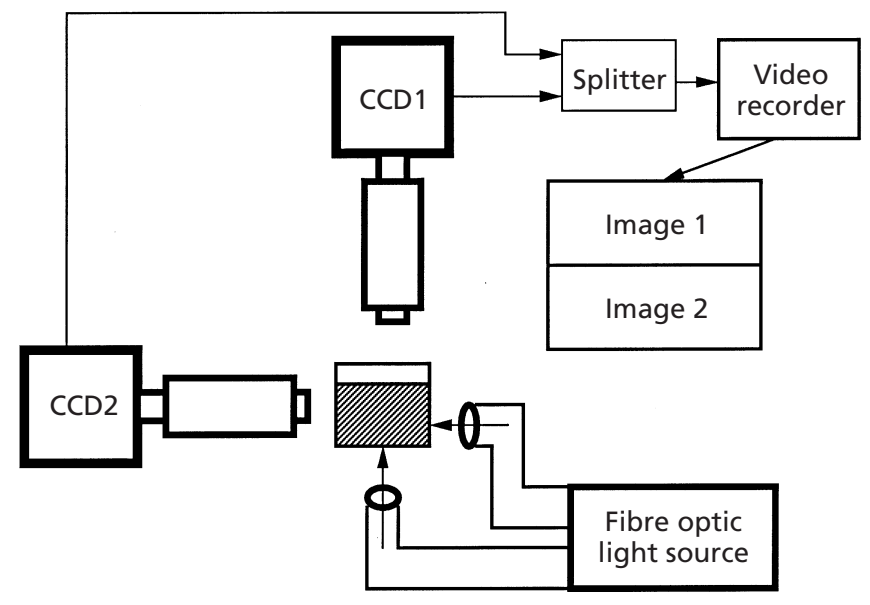

Fig. 1. Diagram showing the design of a dual-view microscope used to obtain images of macrofibre motions on a glass surface. The central glass growth chamber permits light to pass through it in two directions as shown by the arrows leaving the fibre optic light pipes. The images captured by two charge-coupled devices (CCDs) are joined in the splitter and sent to a time-lapse video recorder that writes a time/date tag on each frame. The joined images appear one above the other as shown.

\& Favre, 1987) was used in static cultures housed either in standard $100 \mathrm{~mm}$ diameter plastic Petri dishes or in glass growth chambers constructed from $75 \times 25 \mathrm{~mm}$ glass microscope slides. The chamber dimensions were $56 \times 25 \times 13 \mathrm{~mm}$ (length, width, height). Right-handed FJ7 fibres were produced by overnight growth in $10 \mathrm{ml} \mathrm{TB}$ containing $50 \mathrm{mM}$ $\mathrm{MgSO}_{4}$ at $20^{\circ} \mathrm{C}$. Left-handed RHX fibres were produced in a similar manner using TB containing $50 \mathrm{mM}\left(\mathrm{NH}_{4}\right)_{2} \mathrm{SO}_{4}$. A single intact fibre was transferred into medium of the same composition in the glass growth chamber on which the top was covered with two glass slides. For Petri dish cultures, a single fibre was disrupted by toothpick transfer into medium of the same composition. Both glass chamber and Petri dish cultures used for studies of growth dynamics were grown on a microscope stage at $24^{\circ} \mathrm{C}$.

Video film production and analysis. Three sorts of time-lapse films were produced: low magnification films showing the view of Petri dish cultures from above; side-view films showing contact of the fibres with the floor of the glass growth chamber; and dual-view films that simultaneously show the view from above and from the side of structures in the glass growth chamber. For side-view films the growth chamber was illuminated at an oblique angle using a series 180 high intensity Fibre-Lite (Dolan-Jenner, VWR Scientific Products). Dualview films were illuminated simultaneously from below and from the side with the same light source using two light pipes.

Dual-view films were obtained using the system shown in Fig. 1. Two Cohu charge-coupled device cameras simultaneously captured images from above and from the side. One camera was fitted to an Olympus SZ-Tr zoom stereo microscope positioned above the growth chamber, the other to a Bausch \& Lomb monocular compound microscope tube aimed horizontally at the growth chamber. Both images were transferred to a Phase Eight screen splitter (Vicon Industries). The synchronized output was then sent to a GYYR time-lapse VHS tape deck (Odetics). Both images were recorded simultaneously on the same film, and a time and date stamp was printed on each frame by the GYYR tape deck. The two 
images on each frame were arranged so that the image from the camera positioned above is displayed on the top half of the frame and that showing the side view appears on the bottom half of the frame.

Motion dynamics and touching-point locations were measured by comparison of individual frames either directly using overlays on a video monitor screen or following transfer to a personal computer using Image Pro Plus software (Media Cybernetics). The Adobe Photoshop program (Adobe Systems) was used to assemble the figures. Graphs were produced and analysed with either Cricket Graph or Psi Plot (Poly Software International).

\section{RESULTS}

\section{Pivoting}

When viewed from above at low magnification, live macrofibres are seen to rotate about a centre usually, but not always, located near their mid-points (Mendelson et al., 2000). Although there may be no physical pivot, this rotation is called pivoting in order to distinguish it from other rotations. Pivoting is a helix-hand-specific motion : right-handed fibres pivot clockwise, left-handed ones anti-clockwise. Pivoting requires fibre twisting with growth and contact with a solid surface that provides friction. On plastic surfaces right-handed FJ7 macrofibres, cultured under the standard conditions where their self-assembly takes place, pivoted at a rate of approximately $3.9^{\circ} \min ^{-1}$ with specimen to specimen variation from 2.7 to $5.7^{\circ} \mathrm{min}^{-1}$ (Table 1 ). The lengthdoubling time for FJ7 fibres under these conditions is

Table 1. Pivoting rates of Bacillus subtilis macrofibres on plastic surfaces

Right-handed FJ7 macrofibres (fibres 1-7) were grown in TB medium containing $50 \mathrm{mM} \mathrm{MgSO}_{4}$ at $24{ }^{\circ} \mathrm{C}, 27 \%$ relative humidity; left-handed RHX macrofibres (fibres 8 and 9) were grown similarly in TB medium containing $50 \mathrm{mM}\left(\mathrm{NH}_{4}\right)_{2} \mathrm{SO}_{4}$. Cultures were contained in $100 \mathrm{~mm}$ diameter plastic Petri dishes and viewed from above. Pivoting angle as a function of time was measured from time-lapse video films.

\begin{tabular}{|lrcc|}
\hline Fibre no. & $\begin{array}{c}\text { Pivoting rate } \\
\left({ }^{\circ} \text { min }^{-1}\right)\end{array}$ & $\begin{array}{c}\text { Total angle } \\
\text { pivoted }\left({ }^{\circ}\right)\end{array}$ & $\begin{array}{c}\text { Goodness of } \\
\text { fit }(\boldsymbol{R})^{*}\end{array}$ \\
\hline 1 & 3.38 & 450 & 0.998 \\
2 & 5.67 & 1170 & 0.990 \\
3 & 3.99 & 720 & 0.996 \\
4 & 3.77 & 630 & 0.997 \\
5 & 2.73 & 810 & 0.999 \\
6 & 4.46 & 270 & 0.999 \\
7 & 3.36 & 720 & 0.998 \\
& Mean, 3.90 & & 0.997 \\
9 & 2.13 & 630 & 0.990 \\
9 & 1.72 & 810 & \\
\hline
\end{tabular}

$* R$ values were obtained from regression fits of plots showing total pivoting angle as a function of time.

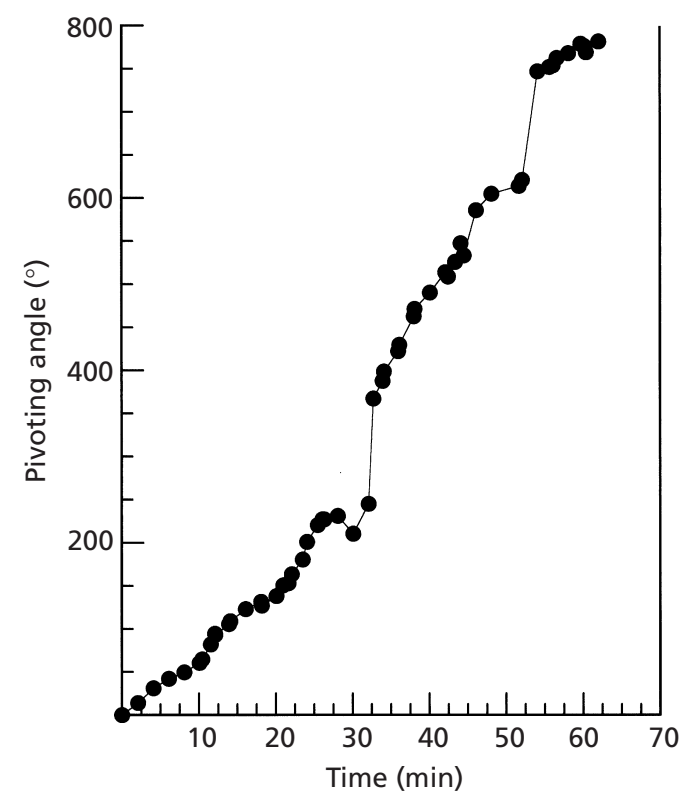

Fig. 2. Pivoting rate of a right-handed FJ7 macrofibre in a glass chamber. The fibre was grown at $24^{\circ} \mathrm{C}$ in TB medium containing $50 \mathrm{mM} \mathrm{MgSO}_{4}$. The data were obtained from the top view images of a time-lapse video film. The angle of the fibre shaft relative to its starting position is plotted as a function of time.

about $90 \mathrm{~min}$ and the twist about 36 turns $\mathrm{mm}^{-1}$. Earlier work has shown that fibres usually supercoil by the time they double their length (Mendelson \& Thwaites, 1990) and that is true also for fibres that are pivoting on a solid surface. All but one of the right-handed fibres underwent folding and winding up of the folded structure during the pivoting motion.

Table 1 also includes measurements of two left-handed structures, fibres 8 and 9, produced by strain RHX which grows with twist about half that of FJ7. Their pivoting rate was also about half that of right-handed FJ7. RHX fibre 9 (Table 1) also folded during the period of observation, and continued to pivot at a constant rate throughout the folding period and for an additional $450^{\circ}$ afterwards. The constancy of pivoting rate shown by the linear regression fits of the data that span folding periods indicates that pivoting is a robust process with a rate that is independent of fibre length but is approximately proportional to twisting rate during growth.

Macrofibre pivoting has been studied at higher magnification on glass surfaces using the growth chambers described earlier. Dual-view films of right-handed FJ7 show that pivoting involves both rolling and stepping over the surface. Rolling is caused by the twisting of a fibre's shaft about its axis as it grows in contact with the surface (a link is available at http://mic. sgmjournals.org for an example of the rolling of a terminal loop described by Mendelson et al., 2000). 

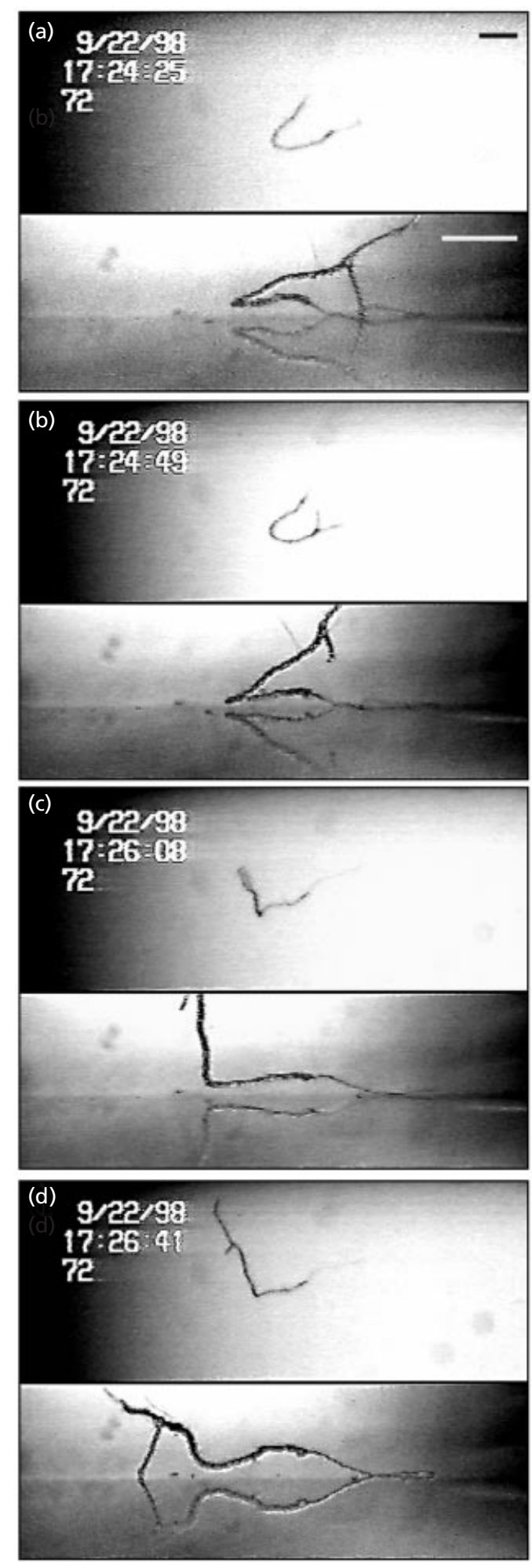

Fig. 3. Contact of a macrofibre with a glass surface. Frames were selected from a time-lapse video film used to produce the data shown in Fig. 2. Panel (a) shows the fibre perched on a side branch near one end and a long thin branch at the other. During the subsequent period, the long thin branch remains in contact, but with changes in how this is distributed. The side branch loses contact and that part of the fibre rotates until it regains contact elsewhere (d), $136 \mathrm{~s}$ later.

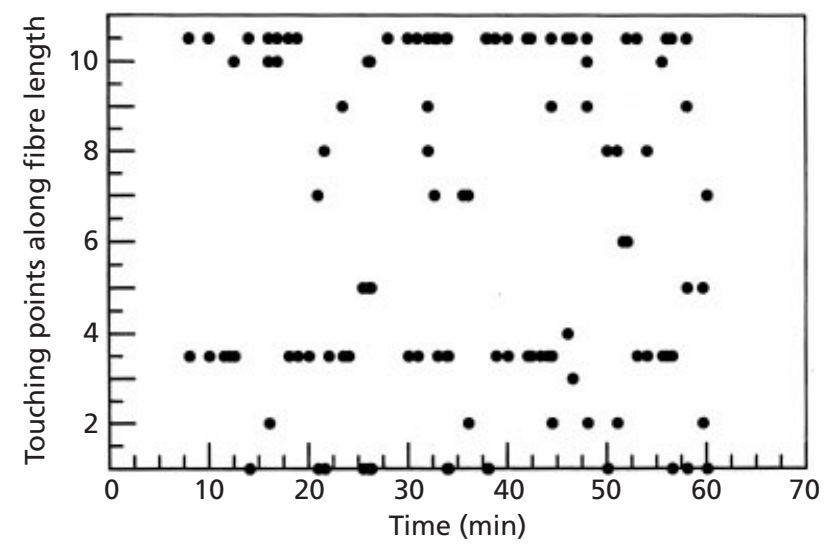

Fig. 4. Locations along macrofibre length that are in contact with a glass surface. Dual view images from video films of the right-handed FJ7 macrofibre of Fig. 2 were used to determine the places where it made contact with the floor throughout a period of growth. Position along the length of the fibre is denoted by stations 1-10 and touching points plotted accordingly. A fibre length greater than 10 indicates a fibre branch near one end that projected beyond the length of the main shaft.

The fibre shaft itself may also rotate about its axis by transferring its weight from one side branch to another as the shaft twists, or by alternating from an elevated position when perched on a branch to lying flat on the surface until twisting brings the branch into contact with the surface again. Such motions take place on a short time scale: that of a single revolution of some section of the macrofibre, i.e. much less than the period of a pivoting revolution. Corresponding variations can be seen in the rate of pivoting (Fig. 2). There are particularly rapid variations at $32.5 \mathrm{~min}$ and at $53 \mathrm{~min}$. The mean pivoting rate from $20 \mathrm{~min}$ onwards is $17.6^{\circ}$ $\min ^{-1}$, so that these rapid variations are separated by almost exactly one revolution of pivoting. There are other smaller short term variations in pivoting rate. Most of these appear however, to be ramifications of the raising and lowering of the fibre shaft as it either perched on short side branches or rested directly on the glass when the branch upon which it had perched was no longer positioned beneath it.

Further examination of dual-view sequences showed that live macrofibres growing on a solid surface did not lie flat on the surface: they were elevated, perching on only a few points along their length. The example of a structure resting on a glass surface shown in Fig. 3 is of the macrofibre considered above. The points of contact with the surface were located where the real (above) and mirror images (below) meet. The horizontal line separating the two images is a projection of the surface. Fig. 3(a) to Fig. 3(d) illustrate that the locations along the fibre length in contact with the surface changed with time. Their distribution has been measured for several different FJ7 macrofibres; Fig. 4 shows the distribution for the macrofibre under consideration. There are two preferred points in contact with the floor much more of 


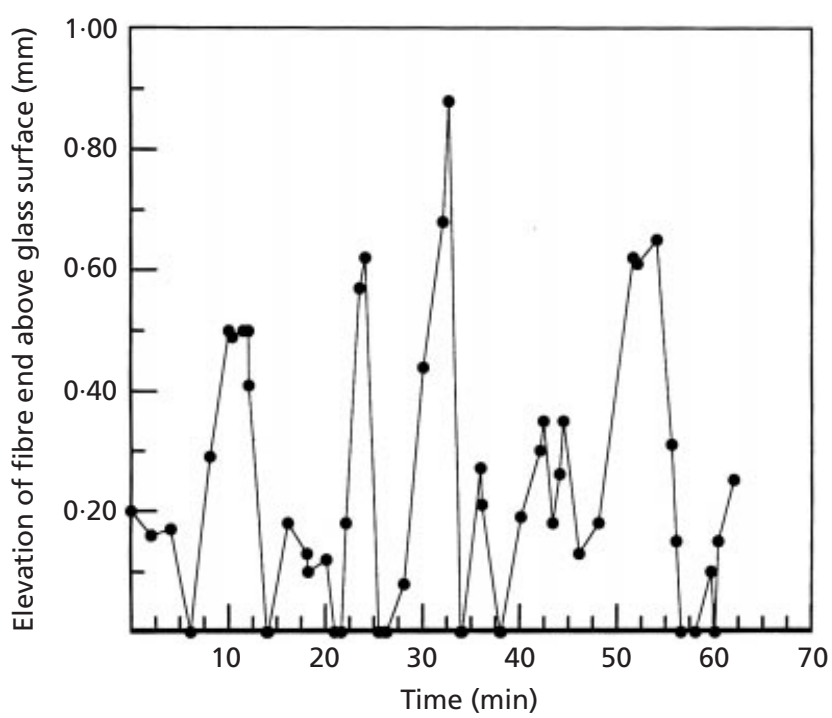

Fig. 5. The elevation of one fibre end of the right-handed FJ7 macrofibre pivoting on a glass surface. The data were measured from dual-view video films from which both pivoting angle and elevation above the surface could be determined.

the time than are other parts of the structure. One such position is located about $1 / 3$ of the total length from one end of the fibre (the 1 end), another beyond the opposite (10) end. The latter contact involves a long branch from the shaft close to the 10 end of the fibre that projects beyond the end of the fibre's shaft. A comparison of the touching point locations found in several fibres shows that preferred locations can exist at many places along a fibre's length. It appears therefore that the distribution of the contact points is governed by the stochastic nature of a macrofibre's development. Nonetheless the mean number of contact points for each fibre is approximately 3.2 and the mean contact time for preferred contacts points is $2 / 3$ of the whole.

The map of fibre contact points with the surface (Fig. 4) in conjunction with other information obtained from side views of the fibre reveals additional details concerning the mechanics of pivoting. i. The centre of pivoting about which the macrofibre moved usually was not in physical contact with the floor. Pivoting throughout much of the sequence was centred between stations 5 and 6 , yet the fibre was rarely in contact with the floor at these locations. ii, There were times when pivoting did revolve around a part of the fibre that was in contact with the floor. One case occurred during the interval between 7 and $12 \mathrm{~min}$. The fibre pivoted $45^{\circ}$ about a centre located at station 3.5 where a branch of the shaft was in contact with the surface throughout this interval. The centre of pivoting eventually shifted back toward the centre of the fibre's length when contact with the floor was lost at station 3.5. iii, Fibre ends were never preferred points of contact throughout the sequence analysed. Their elevation above the surface was correlated however with variations in the rate of pivoting.

Fig. 5 shows the variation with time in the elevation of the 1 end of the macrofibre of Fig. 4. Comparison of Figs 5 and 2 reveals that the major variations in pivoting rate occurred at times when the 1 end took large steps from the floor.

\section{Taking large steps}

In addition to the rotational motions of pivoting, local translational motion of growing macrofibres occur when part of a fibre, usually at an end, rises off the surface and sweeps through an arc before returning to the surface at
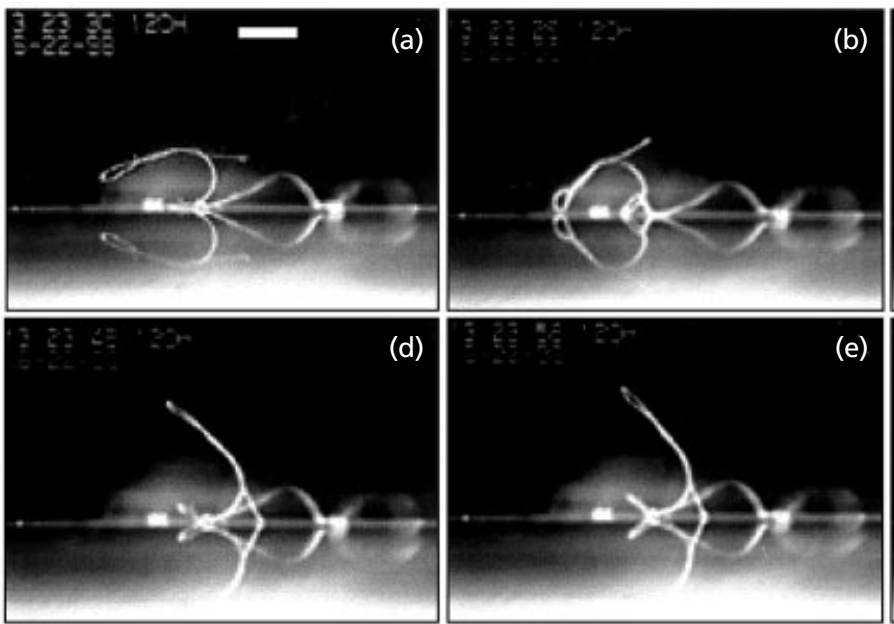

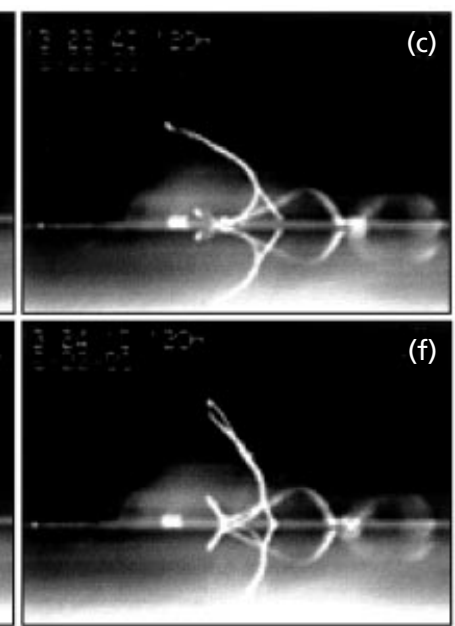

Fig. 6. Side-view images of a right-handed FJ7 macrofibre taking a large step on a glass surface. The fibre was growing in TB medium plus magnesium at $24^{\circ} \mathrm{C}$. A projection of the glass surface appears as a white horizontal line. The fibre image is above; a mirror-image reflection appears below the surface line. Places where the two images meet at the horizontal line are where the fibre touches the floor. The images were transferred from a time-lapse video film into a computer and processed to enhance contrast. Part of the fibre towards the right lies out of focus towards the back of the growth chamber. Time of each frame (s) relative to frame (a), 0; (b), 5; (c), 10; (d), 15; (e), 25; (f), 40. Bar, $0.5 \mathrm{~mm}$. 


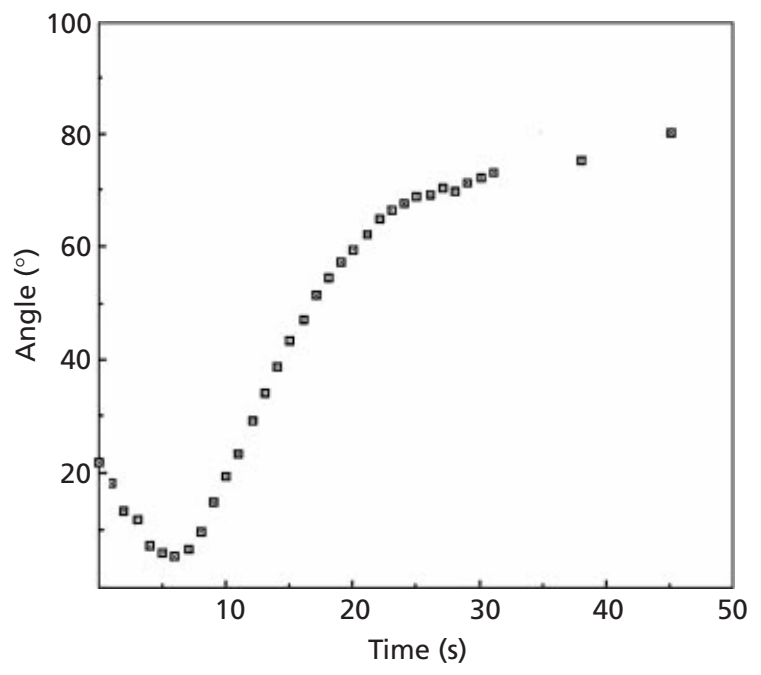

Fig. 7. The rate of rotation of an arm of the right-handed FJ7 macrofibre of Fig. 6 lifting off the floor and sweeping from left to right while undergoing a large walking step. The measurements were made from a side-view video film. The centre about which the motion was measured was located where the stationary part of the fibre made contact with the glass surface. The rate of motion during the initial phase was $4 \cdot 5^{\circ} \mathrm{s}^{-1}$.

a new place. The centre of mass of the fibre is thus displaced. To take a large step, part of the fibre must remain in contact with the surface. This is shown for a different fibre in Fig. 6, in which the terminal loop above the floor in Fig. 6(a) touched the floor in Fig. 6(b), then rose off the surface by the time shown in Fig. 6(c). A portion of the fibre shaft contacting the floor towards the right of Fig. 6(a), however, remained on the surface throughout the entire time span illustrated. There was also a branch protruding from the fibre shaft located towards the centre of the structure shown in Fig. 6(a) that was located above the floor. It made contact with the surface by the time shown in Fig. 6(c) and remained on the surface thereafter. The same kind of branch behaviour can be seen in Fig. 3 .

The rate at which fibres move through the growth medium during large steps has been measured for several steps and used to calculate the torque needed to drive stepping motions (Mendelson et al., 2000). Measurements of the upward sweep of the fibre step illustrated in Fig. 6 show that the fibre arm rose from touching the floor to standing nearly vertical in approximately $20 \mathrm{~s}$ (Fig. 7). The initial decreasing value of angle in Fig. 7 reflects the fact that the fibre tip moved downwards to make contact with the floor before the large upward motion began (see Fig. 6a and b). The rate of rotation during the rise portion of the curve was remarkably constant at $4.5^{\circ} \mathrm{s}^{-1}$, about 15 times faster than the pivoting rate measured on a glass surface using the data from Fig. 2. The rate at which the fibre tip moved during the rise portion was $0.046 \mathrm{~mm} \mathrm{~s}^{-1}$, which corresponds to a chord length of $0.59 \mathrm{~mm}$. The arc length of the moving arm was $0.73 \mathrm{~mm}$.

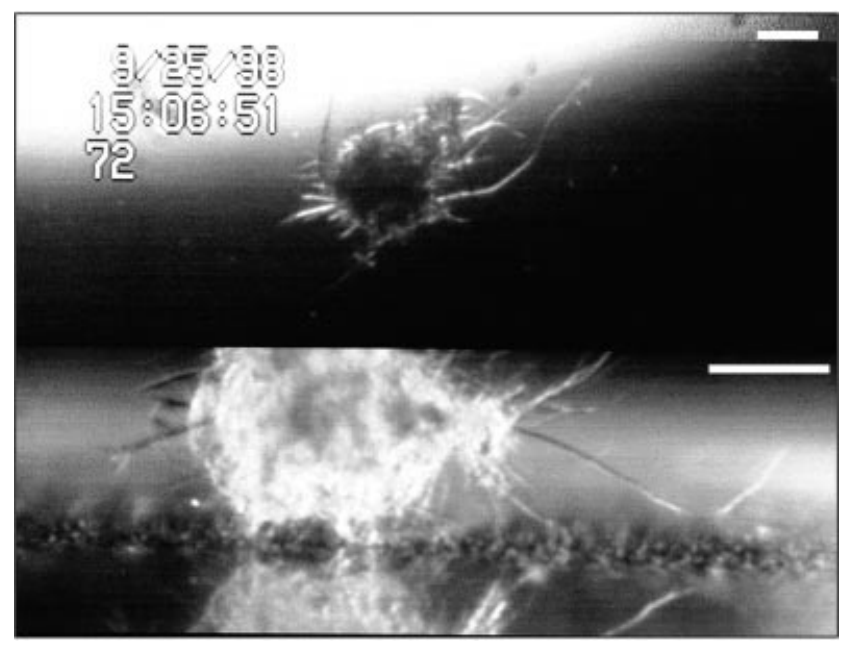

Fig. 8. Above and side view images of a $B$. subtilis macrofibre ball structure showing fibres protruding from its periphery in contact with the floor of the growth chamber. A right-handed fibre was grown in TB medium containing magnesium at $24{ }^{\circ} \mathrm{C}$ in a glass growth chamber and allowed to develop into a ball structure. A dual-view video film showing fibre motions was produced. The large fibre protruding from the surface of the ball towards the right in this frame repeatedly struck the floor and caused the ball structure to pivot. Bars, $0.5 \mathrm{~mm}$.

The asymptotic portion of the curve in Fig. 7 beginning at about $25 \mathrm{~s}$ was caused by twisting of the fibre arm before the upright position was reached. The fibre tip moved through an arc beginning and ending on the floor. The rotation of the tip about the fibre axis could be detected during the sweep but could not be accurately measured in this fibre.

\section{Moving large structures}

Bacterial macrofibres that matured into ball-form structures (Mendelson et al., 1997) also perched on short macrofibre legs that grew from the ball surface (Fig. 8; links to supplementary data for this figure are available at http://mic.sgmjournals.org) The legs supporting these structures twisted and slid over the surface but they were unable in general to cause the entire structure to move. When, however, long fibres grew out of the surface of a ball structure and made contact with the floor, they were seen to pivot about the point of attachment. Motions produced by protruding fibres were observed to cause ball structures to pivot. Pivoting of the larger structure appears to result either from dragging by the pivoting fibre or in smaller structures because the torque on the pivoting fibre due to the drag of the fluid medium is reacted on the ball causing it to rotate in the same direction. (Fig. 9; links to supplementary data for this figure are available at http://mic.sgmjournals.org) Peripheral fibres eventually supercoiled and collapsed onto the ball surface, thereby contributing to its mass. 


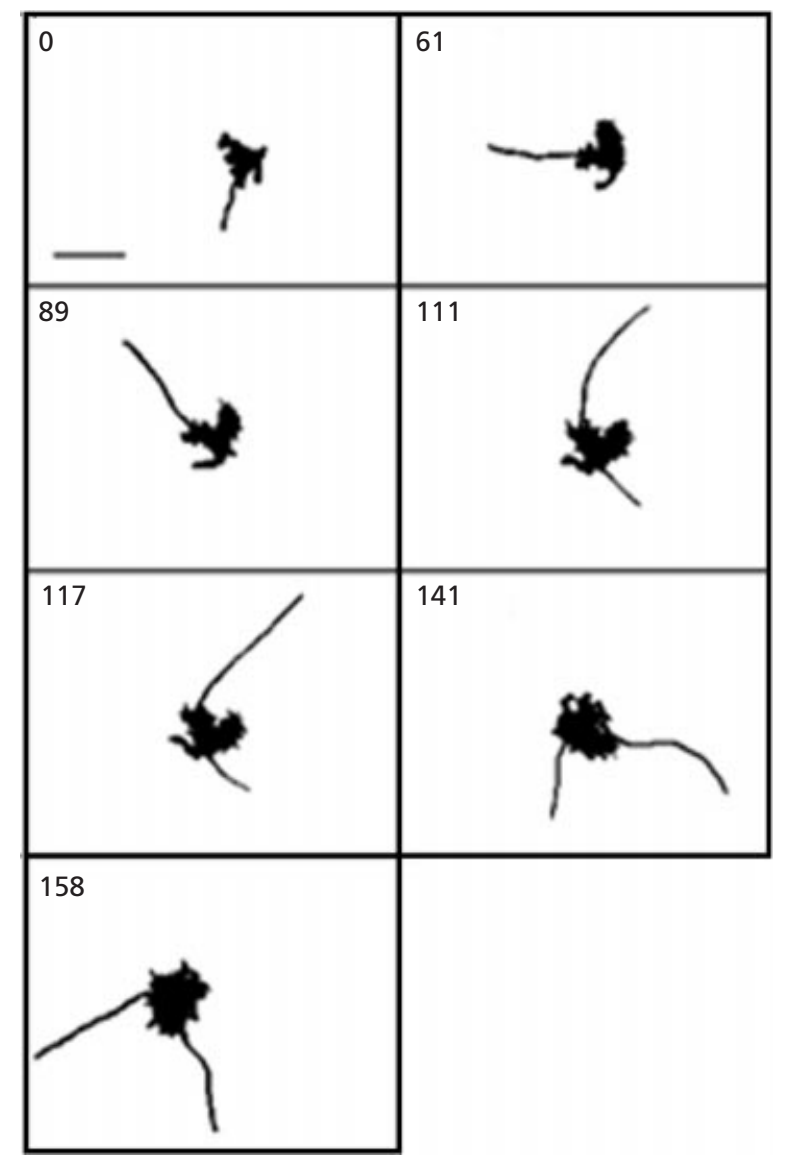

Fig. 9. Pivoting of a ball structure bearing peripheral fibres on a plastic surface. Two peripheral fibres grew out of a righthanded FJ7 macrofibre during the period examined. The shape of the central ball mass serves as a marker indicating that the mass itself pivots. The proximal ends of the fibres are anchored in the ball structure and do not rotate. The distal ends rotate as the fibres elongate and pivot. The video film from which these images were obtained shows that pivoting of the peripheral fibres and the central mass are not closely synchronized. The overall pivoting rate was about $2^{\circ} \mathrm{min}^{-1}$. Pivoting of the ball mass appears to be driven in part as a response to rotation of the peripheral fibre rather than to pivoting of the peripheral fibre about the ball. Times shown are minutes after the initial frame. Bar, $1.0 \mathrm{~mm}$.

\section{DISCUSSION}

Macrofibres undergo morphogenesis in contact with the floor of the chamber in which they grow rather than in suspension as previously thought (Mendelson, 1992; Mendelson et al., 1997). They do so because they are slightly more dense than the growth fluid. Touching a solid surface impedes twisting and may even initiate the writhing motion that leads to fibre-fibre contact, supercoiling and fibre morphogenesis. The newly discovered pivoting of fibres as they grow on a solid surface is shown here to progress at a constant mean rate and to depend on macrofibre twist, but not on macrofibre length. This can be predicted on theoretical grounds, as follows. The ends of a cylinder that twists as it elongates rotate about the axis in opposite directions relative to one another. There is a section midway along the length that does not rotate at all and the rate of rotation increases uniformly with distance from this centre, being $1 / 2 N L^{\circ}$ at the ends, where $N$ is the twist and $L^{*}$ is the fibre growth rate $\left(L\right.$ is the fibre length; $\left.L^{\cdot} \equiv \mathrm{d} L / \mathrm{d} t\right)$. If friction is sufficient to induce rolling or stepping at all sections, then each moves transversely at a speed proportional to distance from the centre; that is the fibre rotates about the centre at an angular speed $N R L^{*} / L$ where $R$ is the cylinder radius. During exponential growth the proportional growth rate $L^{\bullet} / L$ for a given macrofibre in a given environment should not vary with time, so the pivoting rate must be proportional to twist but independent of length.

A simple twisting cylinder is not an ideal model however for a mature macrofibre. Macrofibres are not at all uniform, contact is made at a few points only (a mean of less than 4), and there is a constantly changing pattern to their distribution (Fig. 4). Quite long branches extend from the fibre shaft, the results of past supercoiling involved in morphogenesis, and the tips of branches are often contact points with the surface below (Fig. 3). As a result, it is not surprising that there are substantial variations in pivoting rate (Fig. 2, Table 1). What is surprising is that the mean pivoting rate remains constant during the changing contact pattern and even during supercoiling itself. Pivoting is a remarkably robust phenomenon.

Local translation of macrofibres is achieved when the position of the centre of mass is changed. This occurs by the sweeping of an arm (Fig. 6) and, more significantly, by supercoiling. Suppose that a uniform fibre supercoils by folding and loop formation at its centre; the centre of mass, in this also the centre of pivoting, moves to a point that was formerly one quarter of the length from one end. Similar situations with perhaps smaller displacements arise in the supercoiling of normal macrofibres, i.e. those that are already non-uniform because of asymmetric supercoiling. Macrofibre motion is linked to cell growth, which in itself is quite slow: a $2 \mathrm{~mm}$ long fibre growing under the conditions described elongates at about $0.3 \mu \mathrm{m} \mathrm{s}^{-1}$. This elongation rate is similar to the rate at which eukaryotic cells move by polymerization of filamentous proteins. A nematode sperm cell crawls at about $1 \mu \mathrm{m} \mathrm{s}^{-1}$ by assembling the major sperm protein into filaments (Italiano et al., 2001; Mahadevan \& Matsudaira, 2000). Macrofibres move by insertion of new cell wall polymers into the fabric of the wall (Thwaites \& Mendelson, 1991) in cells throughout the entire fibre.

Although macrofibre movement by growth extension alone is slow in comparison to the rates at which bacterial cells can move using other motility mechanisms, macrofibres can move at speeds approaching those of single cell swimming or gliding (Amsler \& Matsumura, 1995; Berg \& Brown, 1972; Henrichsen, 1972; Macnab, 1996). Rapid speeds in macrofibres are achieved because the collective growth behaviour of all 
the cells within a single macrofibre contribute to its motion and the magnitude of forces resulting from their helical growth is much in excess of that needed to overcome resistances such as viscous drag of the fluid and friction with a solid surface. A $2 \mathrm{~mm}$ long macrofibre pivoting at $17.6^{\circ} \mathrm{min}^{-1}$, for example, has a tip speed of only $5 \mu \mathrm{m} \mathrm{s}^{-1}$, but the tip speed of the moving arm during the step (Figs 6 and 7) is $46 \mu \mathrm{m} \mathrm{s}^{-1}$. The distances traversed by an individual macrofibre are much shorter however than those travelled by cells using conventional motility.

Touching a solid surface during growth strongly influences macrofibre motion and may also play a major role in macrofibre morphogenesis. It is difficult to estimate the magnitude of the friction forces involved. The density of macrofibres is only about $1.015 \mathrm{~g} \mathrm{ml}^{-1}$ so that, even if the whole weight of a fibre were borne by one surface contact, the normal load would be small. For a fibre of $30 \mu \mathrm{m}$ diameter and length $2 \mathrm{~mm}$ it would be a mere $0.2 \mathrm{nN}$, in which case friction could hardly exceed $0 \cdot 1 \mathrm{nN}$. However, the normal force at a contact also depends on the geometry of the macrofibre when bent, as bent beams react substantial forces at their supports. The friction forces may be as large as $1 \mathrm{nN}$. If such a friction force were applied to the tip of a branch such as that making contact in Fig. 3(a and d), which is about $400 \mu \mathrm{m}$ long, the torque transmitted to the macrofibre 'shaft' would be $0.4 \mathrm{pNm}$. If this were applied to a macrofibre of $30 \mu \mathrm{m}$ diameter, the torsional rigidity of which is estimated to be $0.056 \mathrm{pNm}^{2}$, it would twist it by about $0.4^{\circ} \mathrm{mm}^{-1}$. In contrast, the fibre grows with a twist of 36 turns $\mathrm{mm}^{-1}$, approximately 30000 times that resulting from friction. Torques reacted on a fibre shaft due to fluid friction (viscosity) are also of the order of $\mathrm{pNm}$ (for example those developed by a sweeping arm as in Fig. 6 and Mendelson et al., 2000). Clearly these estimated torques are only a tiny fraction of those required to fully block the twisting with growth that is presumed to be the prime cause of macrofibre morphogenesis. The mechanism by which contact with a solid surface influences macrofibre morphogenesis remains therefore to be elucidated. The forces associated with these estimated torques (of the order of $\mathrm{nN}$ ) can however be measured using specially designed cantilever transducers. This is the subject of current research.

The inferred magnitude of forces and torques associated with growth of macrofibres on a solid surface suggest that only a tiny fraction of the potential power of growth is used in macrofibre motion and morphogenesis. The full power available can be estimated as follows. If the twisting with growth of a (cylindrical) macrofibre of torsional rigidity $\mathrm{C}$ and twist $\mathrm{N}$ is resisted by a torque $Q$, the relative (angular) velocity of the ends is $L^{\cdot}(N-Q / C)$ and the power transferred to the resisting load is $Q$ times this. The maximum power transfer is (when $Q=1 / 2 C N$ ) $1 / 4 C L^{*} N^{2}$. For the macrofibre considered above (diameter $30 \mu \mathrm{m}$, length $2 \mathrm{~mm}$ and twist 36 turns $\mathrm{mm}^{-1}$ ) this is approximately $200 \mathrm{pW}$. For comparison, the power produced by a flagellar motor when fully energized is about $2 \times 10^{-3}$
pW (Berg, 1995), and that of a pilus retracting (and consequently causing cells to move over a glass surface) is about $8 \times 10^{-5} \mathrm{pW}$ (Merz et al., 2000). The difference between macrofibre power and that of these other two bacterial motion engines, factors of $10^{5}$ and $10^{8}$ respectively, is very great, but note that a macrofibre contains approximately $5 \times 10^{5}$ cells. This cellular concentration brings the macrofibre's growth power within the realm of possible uses. Twist and writhe can transduce the power of macrofibre growth creating a miniature machine that does work by moving objects. Some examples can be seen in Mendelson (1999) and in sequence 1 at http://research.biology.arizona.edu/ mendelson/enviromicro. Whether such motion serves an adaptive purpose for macrofibres remains to be determined.

\section{ACKNOWLEDGEMENTS}

This work was supported by a grant from the National Center for Research Resources, NIH to N.H.M. J.E.S. was supported by the University of Arizona Undergraduate Biology Research Program. We thank S. D. Whitworth for technical assistance, and K. M. Williams and M. Wagenheim for help in making the video film sequences available on the internet.

\section{REFERENCES}

Amsler, C. D. \& Matsumura, P. (1995). Chemotactic signal transduction in Escherichia coli and Salmonella typhimurium. In Two-Component Signal Transduction, pp. 89-103. Edited by J. A. Hoch \& T. J. Silhavy. Washington, DC: American Society for Microbiology.

Berg, H. C. (1995). Torque generation by the flagellar rotary motor. Biophys J 68, S163-S176.

Berg, H. C. \& Brown, D. A. (1972). Chemotaxis in Escherichia coli analyzed by three-dimensional tracking. Nature 239, 500-504.

Fein, J. E. (1980). Helical growth and macrofiber formation in Bacillus subtilis 168 autolytic enzyme deficient mutants. Can J Microbiol 26, 330-337.

Henrichsen, J. (1972). Bacterial surface translocation: a survey and a classification. Bacteriol Rev 36, 478-503.

Macnab, R. M. (1996). Flagella and chemotaxis. In Escherichia coli and Salmonella: Cellular and Molecular Biology, pp. 123-145. Edited by F. C. Neidhardt and others. Washington, DC: American Society for Microbiology.

Mahadevan, L. \& Matsudaira, P. (2000). Motility powered by supramolecular springs and ratchets. Science 288, 95-99.

Mendelson, N. H. (1976). Helical growth of Bacillus subtilis: a new model of cell growth. Proc Natl Acad Sci U S A 73, 1740-1744.

Mendelson, N. H. (1978). Helical Bacillus subtilis macrofibers: morphogenesis of a bacterial multicellular macroorganism. Proc Natl Acad Sci U S A 75, 2478-2482.

Mendelson, N. H. (1982). Dynamics of Bacillus subtilis helical macrofiber morphogenesis: writhing, folding, close packing, and contraction. J Bacteriol 151, 438-449.

Mendelson, N. H. (1992). Self-assembly of bacterial macrofibers: a system based upon hierarchies of helices. Mat Res Soc Symp Proc 255, 43-54.

Mendelson, N. H. (1999). Bacillus subtilis macrofibers, colonies 
and bioconvection patterns use different strategies to achieve multicellular organization. Environ Microbiol 1, 471-477.

Mendelson, N. H. \& Favre, D. (1987). Regulation of Bacillus subtilis macrofiber twist development by ions: effects of magnesium and ammonium. J Bacteriol 169, 519-525.

Mendelson, N. H. \& Thwaites, J. J. (1988). Studies of Bacillus subtilis macrofiber twist states and bacterial thread biomechanics: assembly and material properties of cell walls. In Antibiotic Inhibition of Bacterial Cell Surface Assembly and Function, pp. 109-125. Edited by P. Actor and others. Washington, DC: American Society for Microbiology.

Mendelson, N. H. \& Thwaites, J. J. (1990). Bending, folding and buckling processes during bacterial macrofiber morphogenesis. Mat Res Soc Symp Proc 174, 171-178.

Mendelson, N. H., Favre, D. \& Thwaites, J. J. (1984). Twisted states of Bacillus subtilis macrofibers reflect structural states of the cell wall. Proc Natl Acad Sci U S A 81, 3562-3566.
Mendelson, N. H., Sahli, B. \& Li, C. (1997). Physical and genetic consequences of multicellularity in Bacillus subtilis. In Bacteria as Multicellular Organisms, pp. 339-365. Edited by J. A. Shapiro \& M. Dworkin. New York: Oxford University Press.

Mendelson, N. H., Sarlls, J. E., Wolgemuth, C. W. \& Goldstein, R. E. (2000). Chiral self-propulsion of growing bacterial macrofibers on a solid surface. Phys Rev Lett 84, 1627-1630.

Merz, A. J., So, M. \& Sheetz, M. P. (2000). Pilus retraction powers bacterial twitching motility. Nature 407, 98-102.

Italiano, J. E., Jr, Stewart, M. \& Roberts, T. M. (2001). How the assembly dynamics of the nematode major sperm protein generate amoeboid cell motility. Int Rev Cytol 202, 1-34.

Thwaites, J. J. \& Mendelson, N. H. (1991). Mechanical behaviour of bacterial cell walls. Adv Microb Phys 32, 173-222.

Received 31 October 2000; revised 5 January 2001; accepted 8 January 2001. 